\title{
THE HUMAN LUMBAR VERTEBRAL BODY AS AN INTRINSIC, FUNCTIONALLY OPTIMAL STRUCTURE
}

\author{
D.N. GHISTA ${ }^{1}$, S.C. FAN ${ }^{2}$, K. RAMAKRISHNA ${ }^{3}$ \& I. SRIDHAR ${ }^{3}$ \\ ${ }^{1}$ School of Chemical and Biomedical Engineering, Nanyang Technological University, Singapore. \\ ${ }^{2}$ School of Civil and Environmental Engineering, Nanyang Technological University, Singapore. \\ ${ }^{3}$ School of Mechanical and Aerospace Engineering, Nanyang Technological University, Singapore.
}

\begin{abstract}
In the vertebral body (VB), the load carrying and transmitting function is primarily performed by the cortical VB Hence, we have modelled the cortical VB as a hyperboloid shell whose geometry and composition are made up of its generators. This paper analyses the forces in the VB generators due to compression, bending and torsional loadings. The unique feature of the hyperboloid geometry is that all the loadings are transmitted as axial forces in the generators. This makes the VB a high-strength structure. Furthermore, because the cortical VB material is primarily made up of its generators (through which all the loadings are transmitted axially), it also makes the VB an intrinsically lightweight structure. We then analyse for the optimal hyperboloid shape and geometry by minimizing the sum of the forces in the hyperboloid VB generators with respect to the hyperboloid shape parameter (angle $\beta$ between pairs of generators). The value of $\beta$ is determined to be $26.5^{\circ}$, which closely matches with the in vivo geometry of the VB based on its magnetic resonance imaging scan. In other words, for the hyperboloid shape parameter $\beta=26.5^{\circ}$, the VB generators' forces are minimal so as to enable it to bear maximal amounts of loadings. In this way, we have demonstrated that the VB is an intrinsically, functionally optimal structure.

Keywords: axial force, bending, toque, generators, hyperboloid, optimal structure, shape parameter, stress analysis, uniaxial compression, vertebral body.
\end{abstract}

\section{INTRODUCTION}

The skeletal system is important to the body both mechanically and metabolically. The bone is the main constituent of the skeletal system and differs from the connective tissues in rigidity and strength. The rigidity and strength of the bone enable the skeleton to maintain the shape of the body, to protect the soft tissues and organs, to supply the framework for the bone marrow, and to transmit the force of muscular contraction from one part of the body to another during movement. The mineral content of the bone serves as a reservoir for ions, particularly calcium, and also contributes to the regulation of extracellular fluid composition, particularly ionized calcium concentration. In addition, the bone is a selfrepairing structural material that is able to adapt its mass, shape and properties to change in mechanical requirements and endures voluntary physical activity for life without breaking or causing pain.

As per the concept of optimal design in nature [1,2], anatomical structures are also customized to be functionally optimal. If it is a load-bearing structure, then it is adroitly designed to be a lightweight and high-strength structure. For example, a long bone is modelled such that it can sustain maximum loading with least material. Consider the case of the femur. Its shape and material density correspond to its stress trajectories under its functional loading (see Fig. 1) as per Wolff's law [3]. In other words, there needs to be less density of bone where the stress trajectories are apart (such as in the trabecular bone) and more density of bone where the stress trajectories are closer (as in the cortical bone).

\subsection{Optimal dimensions of the femur cortical bone}

Now, let us examine the diaphysial part of a long bone (e.g. the femur) that carries most of the bending stress. Using the Euler-Bernoulli flexural equation, the normalized bending strength (BS) 


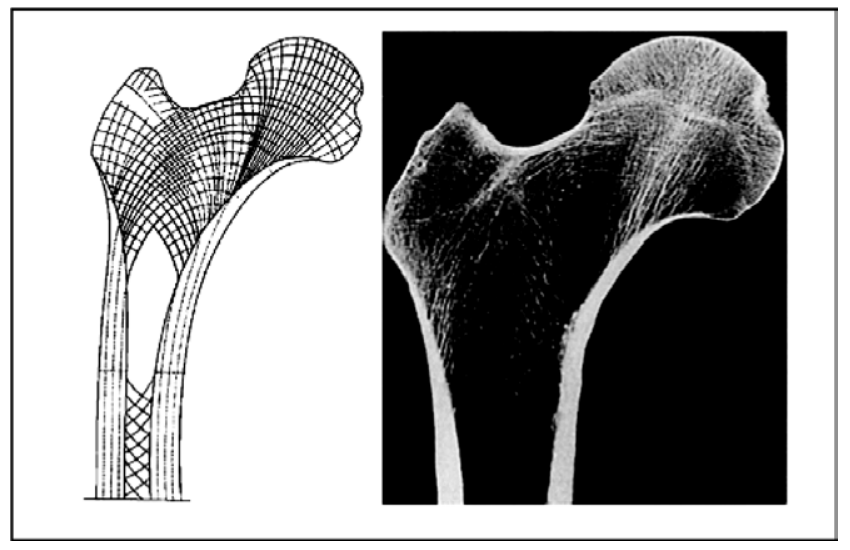

Figure 1: Wolff's drawing of the trabecular orientation in the proximal part of the femur and the cross section of the femur. It can be noted that the bone material distribution corresponds to the orientation of the stress trajectories [3].

can be defined as

$$
\mathrm{BS} \equiv \frac{4 M}{\pi \sigma_{\mathrm{b}} r_{\mathrm{e}}^{3}}=1-\left(\frac{r_{\mathrm{i}}}{r_{\mathrm{e}}}\right)^{4},
$$

where $M$ is the moment on the bone, $\sigma_{\mathrm{b}}$ is the maximum stress on the bone induced due to $M, r_{\mathrm{e}}$ is the external radius of the bone and $r_{\mathrm{i}}$ is the internal radius of the bone (considering the long bone to be a hollow cylinder). Here, normalization is carried out with respect to the moment carrying capacity of a long bone.

Moreover, the normalized weight factor (WF, i.e. normalized weight per unit length) of a long bone can be represented as

$$
\mathrm{WF} \equiv \frac{\pi\left(r_{\mathrm{e}}^{2}-r_{\mathrm{i}}^{2}\right)}{\pi r_{\mathrm{e}}^{2}}=1-\left(\frac{r_{\mathrm{i}}}{r_{\mathrm{e}}}\right)^{2} .
$$

Maximizing the function (BS - WF) with respect to $r_{\mathrm{i}} / r_{\mathrm{e}}$, the optimal $r_{\mathrm{i}} / r_{\mathrm{e}}$ is found to be $1 / \sqrt{2}$ $(=0.707)$, at which the long bone has minimum weight and maximum bending strength; the corresponding area ratio (inner to outer cross-sectional area) is 0.5. Based on our measurements of femur diaphysial cross-sections, this area ratio is in the range $0.5 \pm 0.2$. This analysis shows that at $r_{\mathrm{i}} / r_{\mathrm{e}}=0.707$, bone has maximum bending stiffness, torsional strength and stiffness for minimal weight.

1.2 The spinal vertebral body as an optimal structure

Let us now consider the spine. It protects the spinal cord, while allowing for a full range of motion of the trunk of the body. Spinal biomechanical efficacy is to a large extent based on the optimal intrinsic designs of the spinal vertebral body (VB) and the disc for load bearing. This makes it possible for all types of loadings to be effectively sustained by the VB. In this paper, we first carry out a stress analysis of how effectively the cortical VB can bear uniaxial compression, bending and torsional loads.

Then the relationship between the dimensions of the VB (based on physiological loading conditions) that makes it to be a functionally optimal (lightweight and high-strength) structure is analysed. 


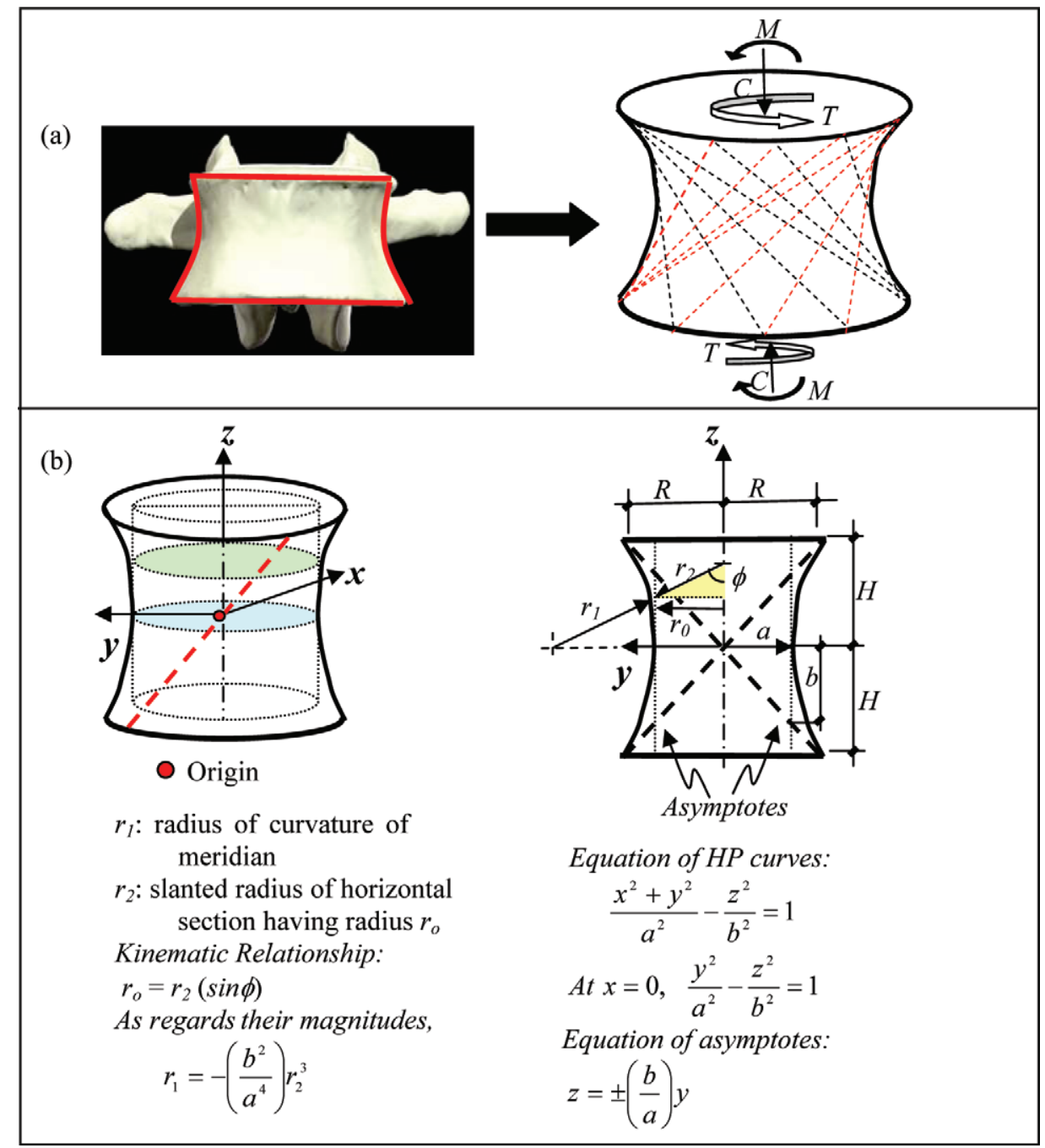

Figure 2: (a) The cortical VB is shaped as a hyperboloid (HP) shell formed of two sets of generators. The height of the HP can be expanded or reduced by the inclination of the generators. It also shows a typical VB. (b) The geometry of the HP shells [4, 5].

In other words, we will provide the relationship between the geometrical parameters of the VB that makes it an intrinsically optimal structure. Finally, the optimal design parameters obtained from the analysis are compared with published magnetic resonance imaging (MRI) scans of VBs.

\section{VB SHAPE AND MEMBRANE STRESSES}

\subsection{Hyperboloid geometry of the VB}

The hyperboloid geometry of the cortical VB is formed by two families of generators, as shown in Fig. 2a. Using shell membrane theory, we will analyse how this hyperboloid VB geometry 
enables the VB to efficiently sustain: (i) compressive loading $C$ on the VB, to cause axial compression in both sets of generators; (ii) bending moment $M$, to result in compressive forces in one set of generators (i.e. on the compression side of the neutral axis) and tensile forces in the other set of generators; (iii) torsional loading $T$, to result in compressive forces (per unit length) in one family of generators and tensile forces in the other family of generators oriented in the other direction.

Figure $2 \mathrm{~b}$ illustrates the hyperboloid geometry of the spinal VB. If we intersect the hyperboloid shell surface with a vertical plane parallel to the $y z$ plane but at $x=-a$, then the intersecting curves will be

$$
\frac{a^{2}+y^{2}}{a^{2}}-\frac{z^{2}}{b^{2}}=1 \quad \text { or } \quad z= \pm\left(\frac{b}{a}\right) y
$$

which has the same slope as the asymptotes. Based on the hyperboloid geometry [4], the hyperboloid surface can be generated by a pair of intersecting lines inclined at an angle $\beta=\tan ^{-1}(a / b)$ in the vertical plane tangent to the waist circle $\left(r_{0}=a\right)$.

The construction of the cortical VB hyperboloid by a set of generators [5] is illustrated in Fig. 3, wherein the end plate radius $\mathrm{AN}$ is $R$, the radius of the waist circle is $a$ and the height of the $\mathrm{VB}$ is $2 H$. Based on this, we define

$$
\tan \beta=\frac{\sqrt{R^{2}-a^{2}}}{H}=\frac{a}{b} .
$$

The primary dimensional parameters of the VB hyperboloid are $(R, a$ and $H)$, and $\tan \beta$ provides the relationship between them.

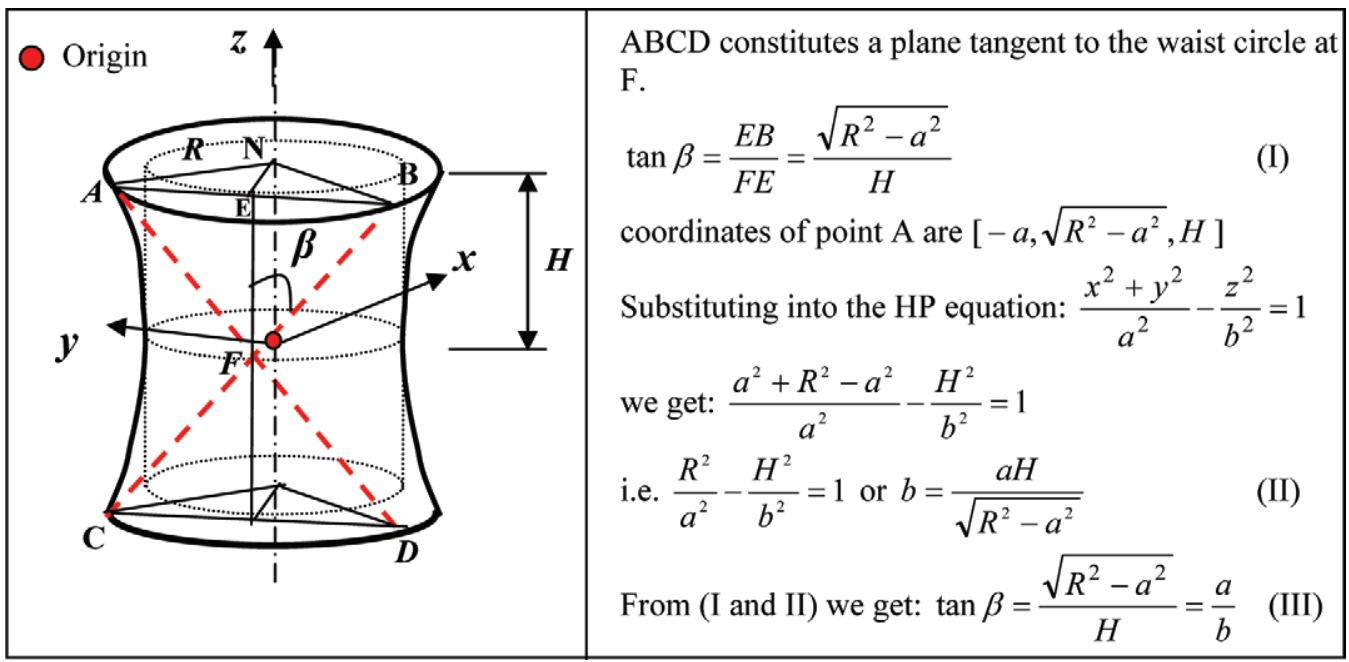

Figure 3: The geometry of hyperboloid generator: the generators $\mathrm{AD}$ and $\mathrm{BC}$ form the basis for the construction of the hyperboloid. 


\subsection{Membrane stresses in the VB cortex}

We follow the membrane theory of shells to analyse the stresses in the cortical VB [6]. Membrane stresses $\sigma_{\phi}$ (along the meridian) and $\sigma_{\theta}$ (along the hoop) have a relationship with the normal pressure $p_{r}$, as depicted in Fig. 4. The equilibrium of forces in the radial $(r)$ direction gives

$$
-2 \sigma_{\phi} t\left(r_{2} \mathrm{~d} \theta\right) \sin \left(\frac{\mathrm{d} \phi}{2}\right)+2 \sigma_{\theta} t\left(r_{1} \mathrm{~d} \phi\right) \sin \left(\frac{\mathrm{d} \theta}{2}\right)+p_{r}\left[2 r_{1} \sin \left(\frac{\mathrm{d} \phi}{2}\right) \cdot 2 r_{2} \sin \left(\frac{\mathrm{d} \theta}{2}\right)\right]=0
$$

wherein, in the case of a hyperboloid, $r_{2}$ is considered to be positive and $r_{1}$ is considered to be negative, and their magnitudes in terms of $a, b$ and $\phi$ are

$$
\begin{aligned}
& r_{1}=\frac{a^{2} b^{2}}{\left(a^{2} \sin ^{2} \phi-b^{2} \cos ^{2} \phi\right)^{3 / 2}}, \\
& r_{2}=\frac{a^{2}}{\left(a^{2} \sin ^{2} \phi-b^{2} \cos ^{2} \phi\right)^{1 / 2}} .
\end{aligned}
$$

For small angles, $\sin \theta \approx \theta$, which leads eqn (5) to

$$
-\sigma_{\phi} t r_{2}(\mathrm{~d} \theta)(\mathrm{d} \phi)+\sigma_{\theta} t r_{1}(\mathrm{~d} \phi)(\mathrm{d} \theta)=-p_{r}\left[r_{1}(\mathrm{~d} \phi) \cdot r_{2}(\mathrm{~d} \theta)\right]
$$

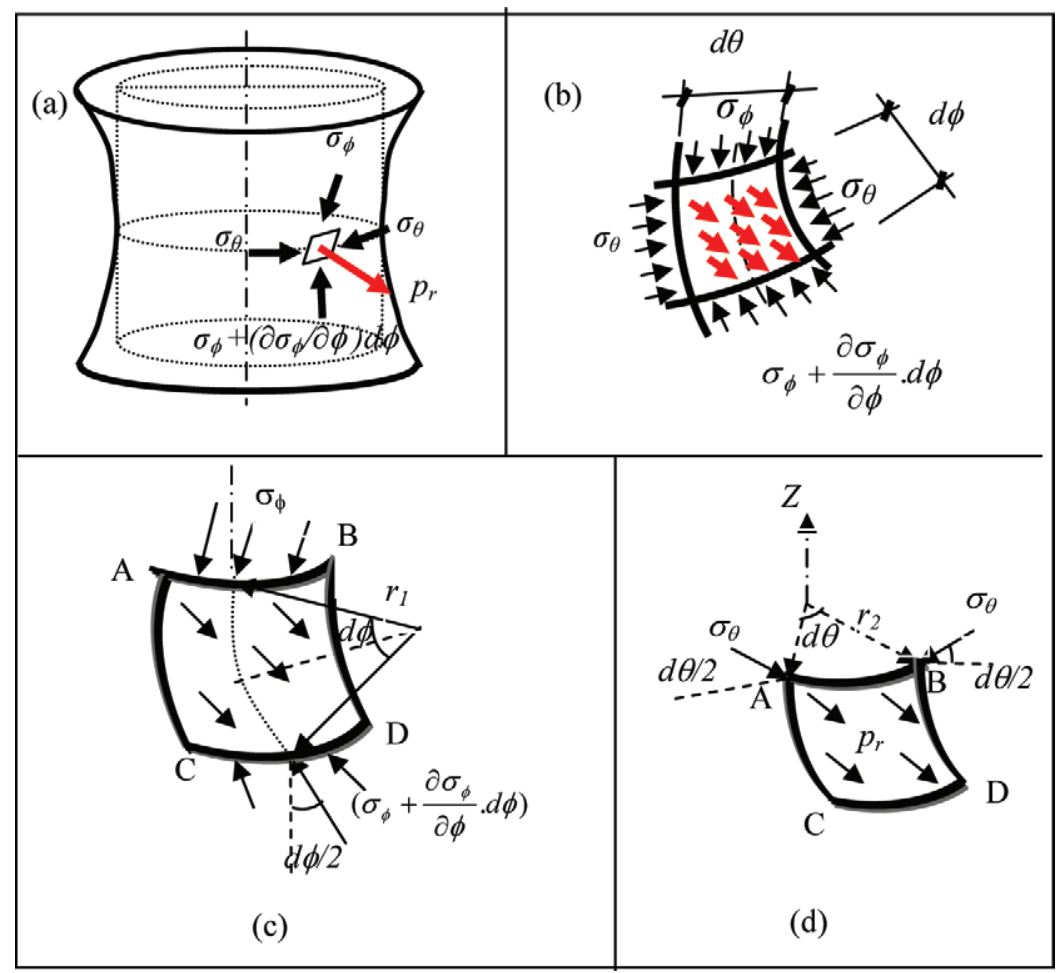

Figure 4: The stresses acting on an element of the hyperboloid shell. 
or

$$
\frac{\sigma_{\phi} t}{r_{1}}-\frac{\sigma_{\theta} t}{r_{2}}=p_{r} .
$$

Denoting $N_{\phi}=\sigma_{\phi} t$ and $N_{\theta}=\sigma_{\theta} t$ as the stresses per unit wall thickness (or stress resultants), with $t$ being the VB wall thickness, we obtain

$$
\frac{N_{\phi}}{r_{1}}-\frac{N_{\theta}}{r_{2}}=p_{r},
$$

which is the membrane equation for the hyperboloid VB shell. This is because, for a hyperboloid shell, $r_{1}$ is negative and $r_{2}$ is positive. Now $p_{r}$ is negligible due to the cancellous bone within the VB cortical shell. Hence, by substituting $p_{r}=0$ (i.e. for an internally non-pressurized cortical VB hyperboloid shell) in eqn (9), we obtain

$$
N_{\phi}=\left(\frac{r_{1}}{r_{2}}\right) N_{\theta} .
$$

Substituting $r_{1}=\left(b^{2} / a^{4}\right) r_{2}^{3}$ from Fig. 2 into eqn (10), we obtain

$$
N_{\phi}=\left(\frac{b^{2}}{a^{4}} r_{2}^{2}\right) N_{\theta} .
$$

\section{ANALYSIS OF FORCES IN THE VB GENERATORS UNDER DIFFERENT LOADINGS}

\subsection{Stress analysis under axial compression}

We will now analyse the stresses in the hyperboloid shell (generators) due to a uniaxial compressive force, as shown in Fig. 5. Assume that there are two sets of $n$ number of straight bars, placed at an equal spacing of $(2 \pi a / n)$ measured at the waist circle, which constitute the hyperboloid surface as shown in Fig. 5b. Due to the axisymmetric nature of the vertical load, no shear stresses are incurred in the shell, i.e. $\sigma_{\phi \theta}=0$ as in Fig. 5a. We then delineate a segment of the hyperboloid shell and consider its force equilibrium (as illustrated in Fig. 5c). At any horizontal section, by force equilibrium

$$
\left(2 \pi r_{0}\right) N_{\phi}(\sin \phi)=C .
$$

Now, consider the segment at the waist circle, where $\phi=90^{\circ}$ and $r_{2}=r_{0}=a$ (throat radius),

$$
(2 \pi a) N_{\phi\left(\phi=90^{\circ}\right)}=C \quad \text { or } \quad N_{\phi\left(\phi=90^{\circ}\right)}=\frac{C}{2 \pi a} \text { (compressive). }
$$

At the waist circle where $r_{2}=a$, eqn (11) yields

$$
N_{\theta\left(\phi=90^{\circ}\right)}=\left(\frac{a^{4}}{b^{2}} \frac{1}{r_{2}^{2}}\right) N_{\phi\left(\phi=90^{\circ}\right)}=\left(\frac{a^{2}}{b^{2}}\right) N_{\phi\left(\phi=90^{\circ}\right)},
$$

which on combining with eqn (13), leads to

$$
N_{\theta\left(\phi=90^{\circ}\right)}=\left(\frac{a^{2}}{b^{2}}\right) \frac{C}{2 \pi a}=\frac{C}{2 \pi a} \tan ^{2} \beta,
$$

which is compressive in nature. 


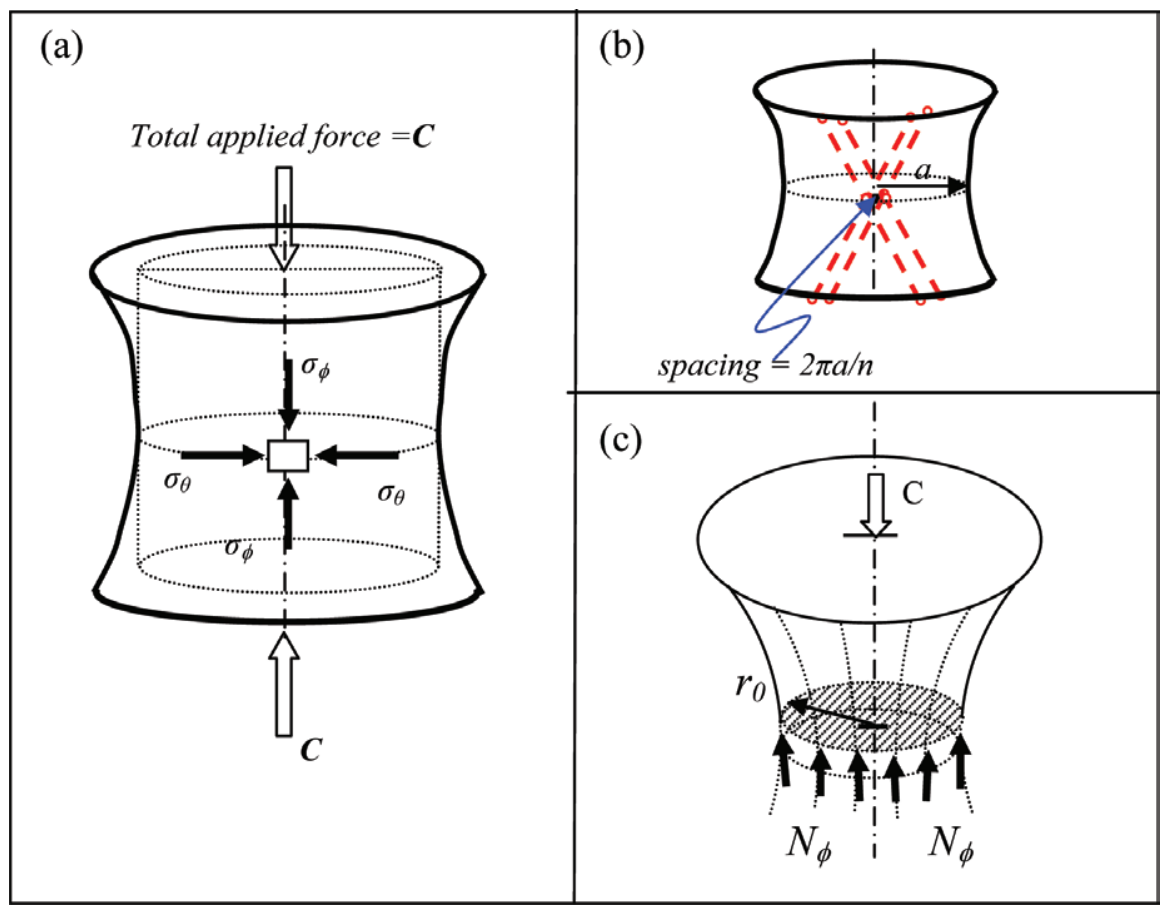

Figure 5: The stresses at the waist section of a hyperboloid shell. (a) Stress components; (b) equivalent straight bars (aligned with the generators) placed at equal spacing to take up the stresses; (c) equilibrium of forces on a shell segment.

From Fig. 6, the equivalent resultant compressive force $F_{c}$ in a fibre (generator of the hyperboloid surface) is given by

$$
F_{\mathrm{c}}^{2}=\left[N_{\phi}\left(\frac{\pi a}{n}\right)\right]^{2}+\left[N_{\theta}\left(\frac{\pi b}{n}\right)\right]^{2} .
$$

Substituting eqns (13) and (14) into eqn (15), we have

$$
F_{\mathrm{c}}=\frac{C}{2 n \cos \beta}=\frac{C \sqrt{H^{2}+R^{2}-a^{2}}}{2 n H} .
$$

Thus the total axial loading is transmitted into the hyperboloid shell's straight generators as compressive forces.

\subsection{VB stress analysis under bending moment}

When the VB is subjected to a bending moment $(M)$, normal stresses $\left(\sigma_{y}\right)$ are developed at the waist circle $\left(r_{0}=a\right)$ cross-section, as shown in Fig. 7. The bending moment sustained at the waist circle is given by

$$
M=2 \int_{0}^{a} \sigma_{y}\left[2 \frac{t}{\cos \alpha} \mathrm{d} y\right] y,
$$




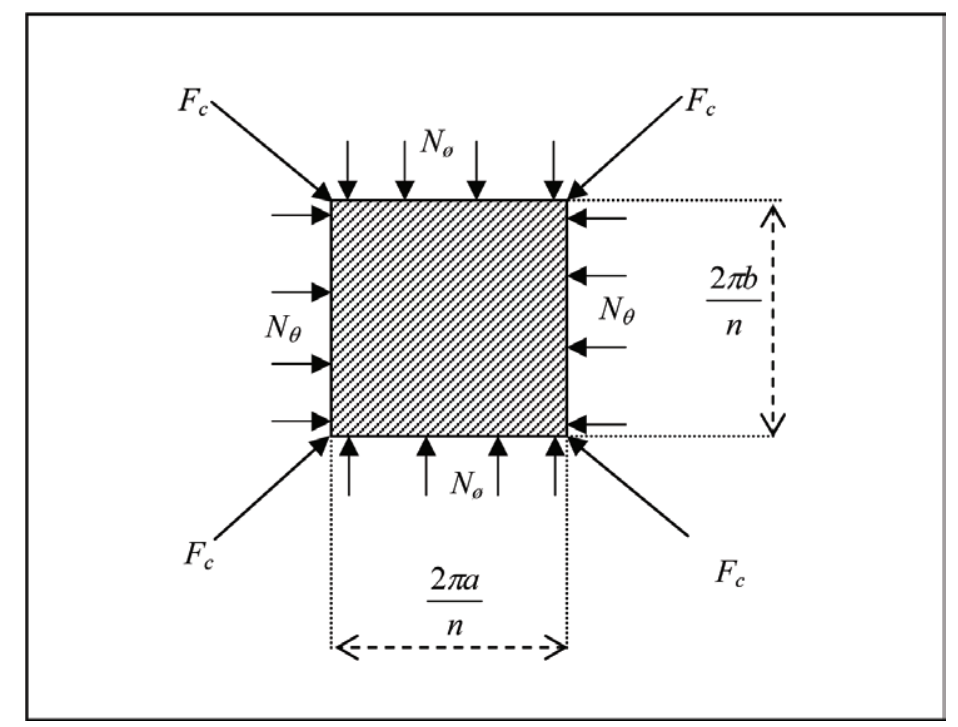

Figure 6: The equivalent diagonal forces in the intersecting bars to take up the stresses around a shell element.

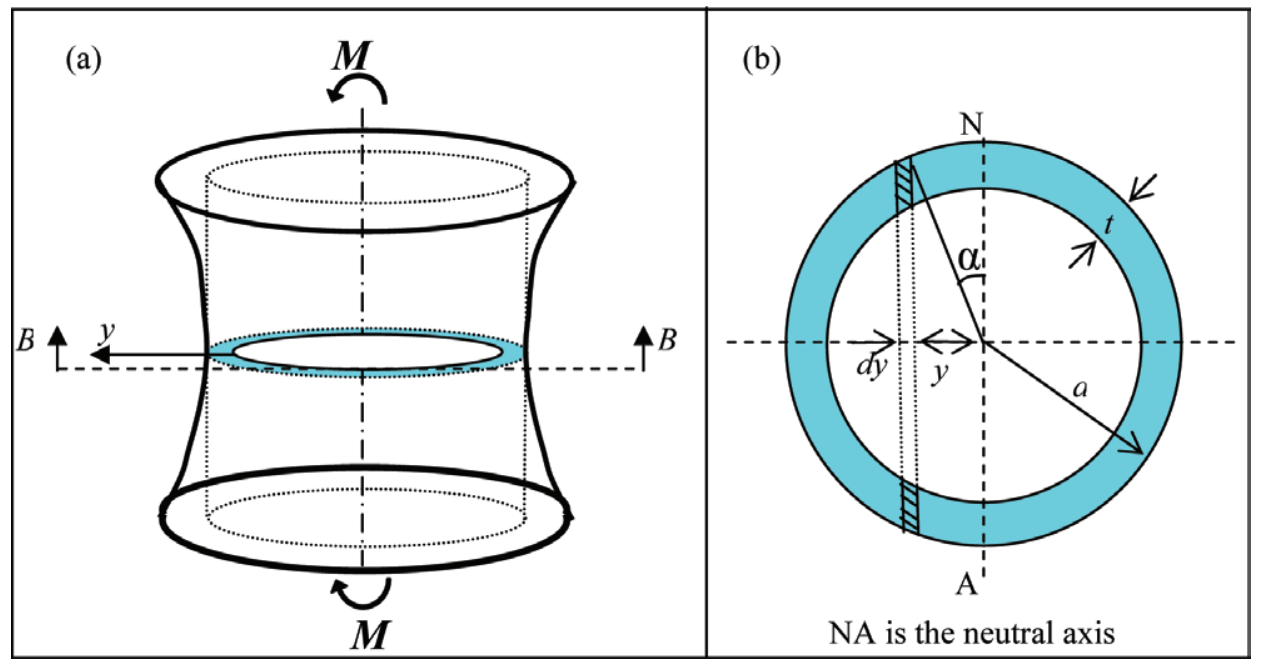

Figure 7: (a) The bending moment on the VB. (b) Plan view at the section BB (i.e. at the waist circle).

where $\sigma_{y}$ is the compressive stress normal to the cross section (due to the bending moment $M$ ) acting on the two rectangular elements of length $2(t / \cos \alpha)$ and width $\mathrm{d} y$. In addition,

$$
\sigma_{y}=\frac{y}{a} \sigma_{a}
$$

where $\sigma_{a}$ is the stress at $y=a$. 
Combining eqns (17) and (18), we have

$$
\begin{aligned}
& M=4 \int_{0}^{a} \frac{y^{2}}{a} \sigma_{a} \frac{t}{\cos \alpha} \mathrm{d} y \\
& M=4 t \sigma_{a} \int_{0}^{a} \frac{y^{2}}{a \cos \alpha} \mathrm{d} y .
\end{aligned}
$$

Substituting for $y=a \sin \alpha$ and $\mathrm{d} y=a \cos \alpha \mathrm{d} \alpha$, eqn (19) can be rewritten as

$$
M=4 t \sigma_{a} \int_{0}^{\pi / 2} \frac{a^{2} \sin ^{2} \alpha}{a \cos \alpha} a \cos \alpha \mathrm{d} \alpha .
$$

Integrating eqn (20) gives

$$
M=\pi a^{2} t \sigma_{a}
$$

The normal stress at the waist circle in terms of the bending moment can be written as

$$
\sigma_{a}=\frac{M}{\pi a^{2} t}
$$

Then, $N_{\phi}$, on the waist-circle element at distance $a$ from the neutral axis, is given by

$$
\left(N_{\phi}\right)_{a}=\sigma_{a} t
$$

Thus from eqn (22),

$$
\left(N_{\phi}\right)_{a}=\frac{M}{\pi a^{2}} .
$$

According to Fig. 8c the force $\left(F_{m}\right)$ in the generator is given by

$$
F_{m}^{2}=\left[\left(\frac{\pi a}{n}\left(N_{\phi}\right)_{a}\right)^{2}+\left(\frac{\pi b}{n}\left(N_{\theta}\right)_{a}\right)^{2}\right] .
$$

Substituting the value of $N_{\theta}$ from eqn (11), we get

$$
F_{m}^{2}=\left[\left(\frac{\pi a}{n}\left(N_{\phi}\right)_{a}\right)^{2}+\left(\frac{\pi b}{n}\left(\frac{a^{2}}{b^{2}}\left(N_{\phi}\right)_{a}\right)\right)^{2}\right] .
$$

Since $\tan \beta=a / b$, eqn (25) reduces to

$$
F_{m}^{2}=\left(\frac{M}{\pi a^{2}}\right)^{2}\left(\frac{\pi a}{n}\right)^{2}\left[1+\tan ^{2} \beta\right]
$$

or

$$
F_{m}=\frac{M}{n a \cos \beta},
$$

where $F_{m}$ can be either compressive or tensile force based on the location of the generators relative to the plane about which the bending moment is applied. 


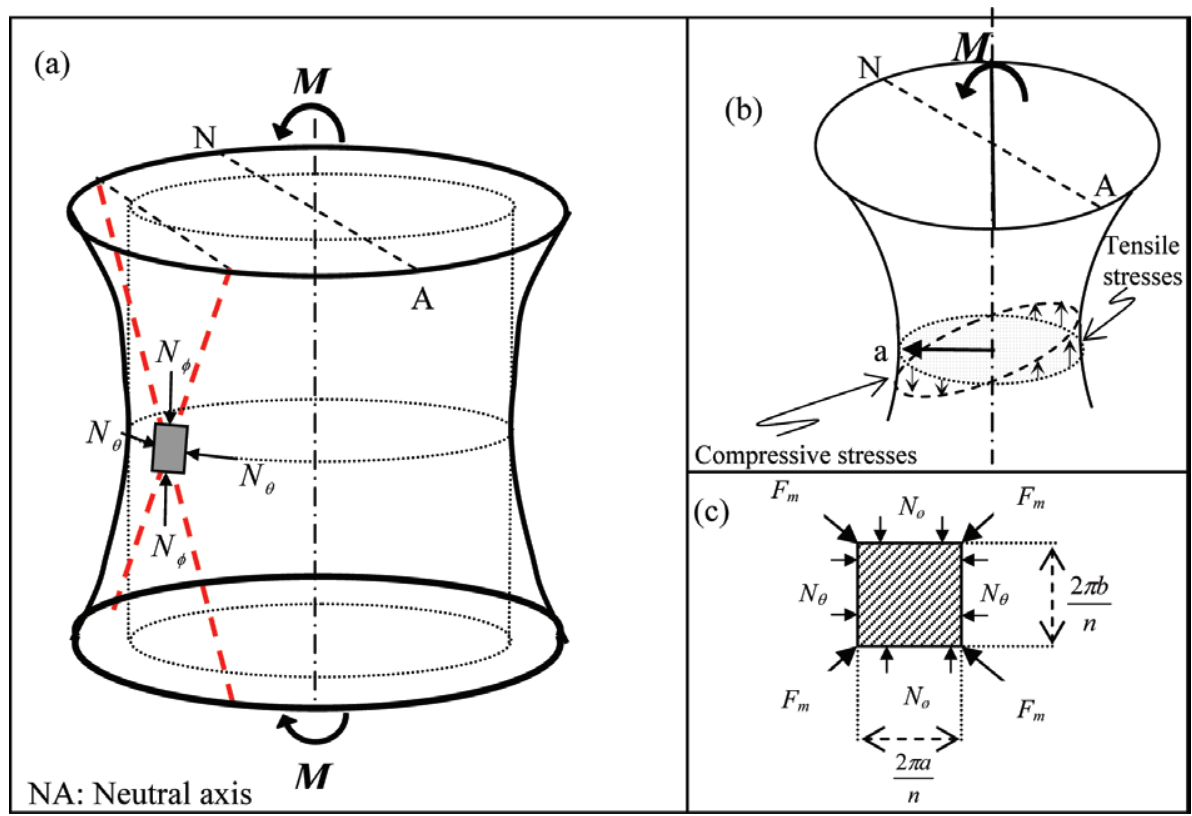

Figure 8: (a) Stress resultants at the waist section of the hyperboloid shell under bending. (b) Equilibrium of forces on a shell segment. (c) Equivalent diagonal forces in the intersecting bars to take up the stresses around a shell element on the compression side of the hyperboloid shell.

\subsection{Stress analysis under torsional loading}

Next, we analyse the compressive and tensile forces in the hyperboloid shell generators when the VB is subjected to pure torsion $(T)$. In this case (refer to Fig. 9a), the normal stress resultants are zero and only have shear resultants

$$
N_{\phi}=N_{\theta}=0 \quad \text { and } \quad N_{\phi \theta}=\tau t .
$$

The equilibrium of a segment of the shell at a horizontal section (as in Fig. 9b) gives,

$$
\left[\left(2 \pi r_{0}\right) N_{\phi \theta}\right] r_{0}=T \quad \text { or } \quad\left(2 \pi r_{0}^{2}\right) N_{\phi \theta}=T
$$

At the waist circle, $r_{2}=r_{0}=a$ (throat radius):

$$
(2 \pi a t \tau) a=T, \quad \text { i.e. } N_{\phi \theta}=\frac{T}{2 \pi a^{2}} .
$$

Now, consider an element at the waist circle as shown in Fig. 9c. The equivalent compressive force $\left(F_{\mathrm{c} T}\right)$ and tensile force $\left(F_{\mathrm{t} T}\right)$, in the direction aligned to their respective set of shell generators, are given by

$$
F_{\mathrm{c} T}^{2}=F_{\mathrm{t} T}^{2}=\left(N_{\phi \theta} \frac{\pi a}{n}\right)^{2}+\left(N_{\phi \theta} \frac{\pi b}{n}\right)^{2}
$$




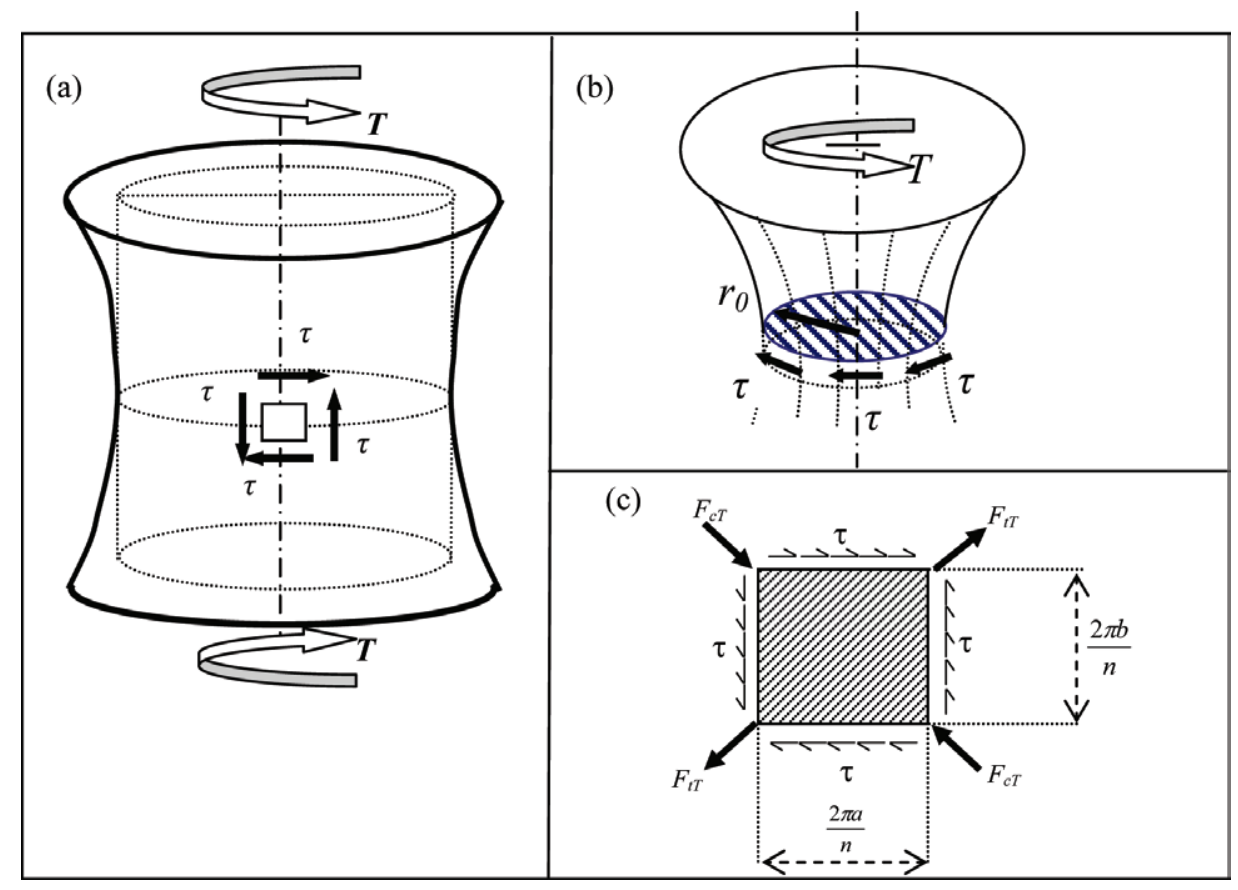

Figure 9: (a) Stress resultants in the hyperboloid shell element $\left(N_{\phi}=N_{\theta}=0\right.$ and $\left.N_{\phi \theta}=\tau t\right)$ due to torsion $T$ acting on the VB. (b) Equilibrium of a shell segment under torsion $(T)$ and shear stresses $(\tau)$ (or shear stress resultant $N_{\phi \theta}$ ). (c) Equivalent diagonal forces in the intersecting generators to take up the stresses around the shell element.

or

$$
\left|F_{\mathrm{c} T}\right|=\left|F_{\mathrm{t} T}\right|=\frac{T}{2 n a \sin \beta} .
$$

Thus, a torsional loading on the hyperboloid shell VB is taken up by one set of generators being in compression and the other set of generators being in tension.

\section{OPTIMAL DESIGN}

\subsection{Structural analogy of the VB to the cane stool}

The above analyses illustrate how the intrinsic hyperboloid shape design of the VB enables the loadings to be transmitted as axial (compressive/tensile) forces through the generators of the hyperboloid shell. In this regard, the VB can be compared to a hyperboloid cane stool (shown in Fig. 10a), which is an ideal high-strength and lightweight structure. This is because all the loading exerted on it (by a person sitting on it) is transmitted (to the ground) as axial forces in the cane generators. A material (such as cane) is strongest in compression provided its length is less than the buckling length. This makes the cane stool a high-strength and high load-bearing structure.

If the two sets of canes (at $\pm \beta$ ) are encircled at the waist circle by a band, it increases the loadcarrying capacity of the cane stool. If, additionally, these two sets of canes $( \pm \beta)$ are tied at all 


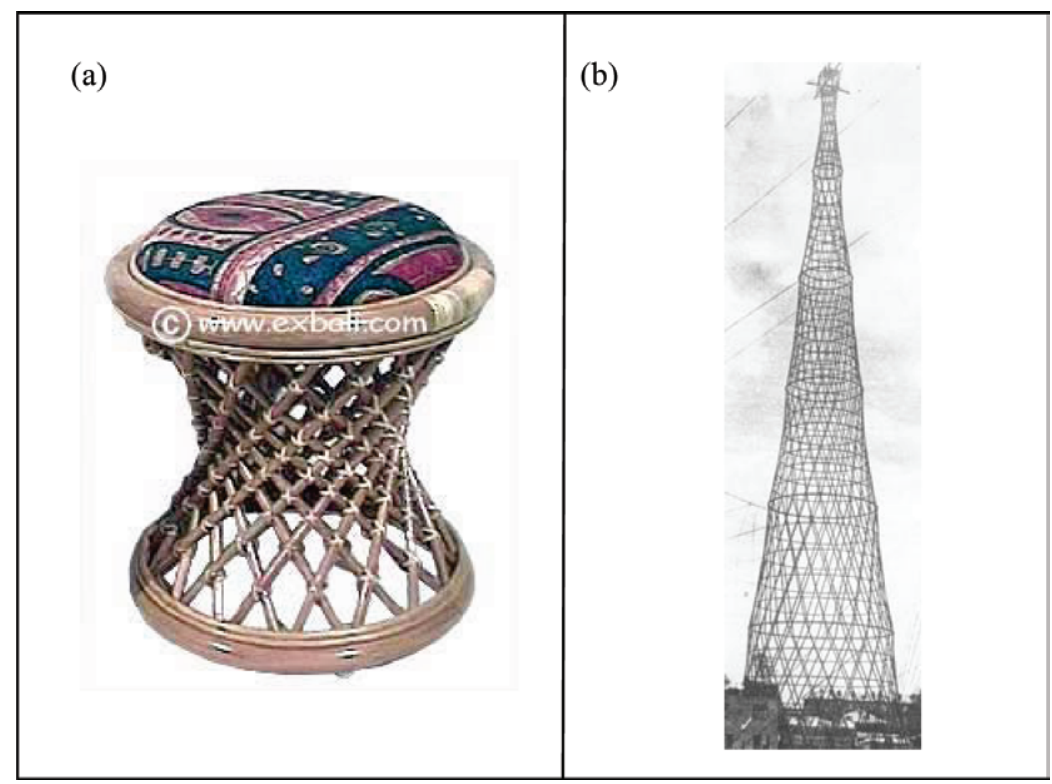

Figure 10: A couple of man-made hyperboloid structures: (a) the humble cane stool (reproduced with permission from www.exbali.com) weighing $2.5 \mathrm{~kg}$ but capable of bearing a load of $5000 \mathrm{~N}$ in compression; (b) the Shabolovka radio tower, Moscow, needed just 2200 tons of steel to build a $350 \mathrm{~m}$ high tower [7].

their intersecting points (as shown in Fig. 10a), their functional lengths are reduced, which further enhances the strength and load-carrying capacity of the cane stool. Furthermore, the cane stool is very light as it is just made up of discrete canes (as generators of the hyperboloid structure). This structural configuration makes the cane stool a very simple but effective load-bearing, high-strength and lightweight structure. Incidentally, such a cane stool of $(R=200 \mathrm{~mm}, H=175 \mathrm{~mm})$ has a nominal weight of $2.5 \mathrm{~kg}$ (or $25 \mathrm{~N}$ ), but it can easily bear a load of at least $5000 \mathrm{~N}$, which is 200 times its weight.

The spinal VB cortex has similar structural configuration and properties as the cane stool, which also make it an efficient load-bearing and load-transmitting, high-strength and lightweight structure. The VB wall can be considered to be primarily comprised of the two sets of generators. Just as in the case of the cane stool and the Shabolovka radio tower (Fig. 10), the VB wall transmits all the loading as axial forces through its generators. This is the basis for a high-strength and lightweight VB design.

\subsection{Optimization of the hyperboloid shape of the VB}

The spinal VB has a definitive value range for the hyperboloid shape parameter $\beta$ and hence for its hyperboloid shape. In order to determine the structural basis of this $\beta$ value, we will calculate that value of $\beta$ which makes the combined axial force in its generators to be minimum. In this case, the optimized VB structure will be able to sustain maximal loading, before the ultimate failure load of its generators is reached.

The VB is subjected to the combined compression, bending moment and torsional loadings. Under this combined loading, the forces in the generators given by eqns (16), (26) and (30) can be 


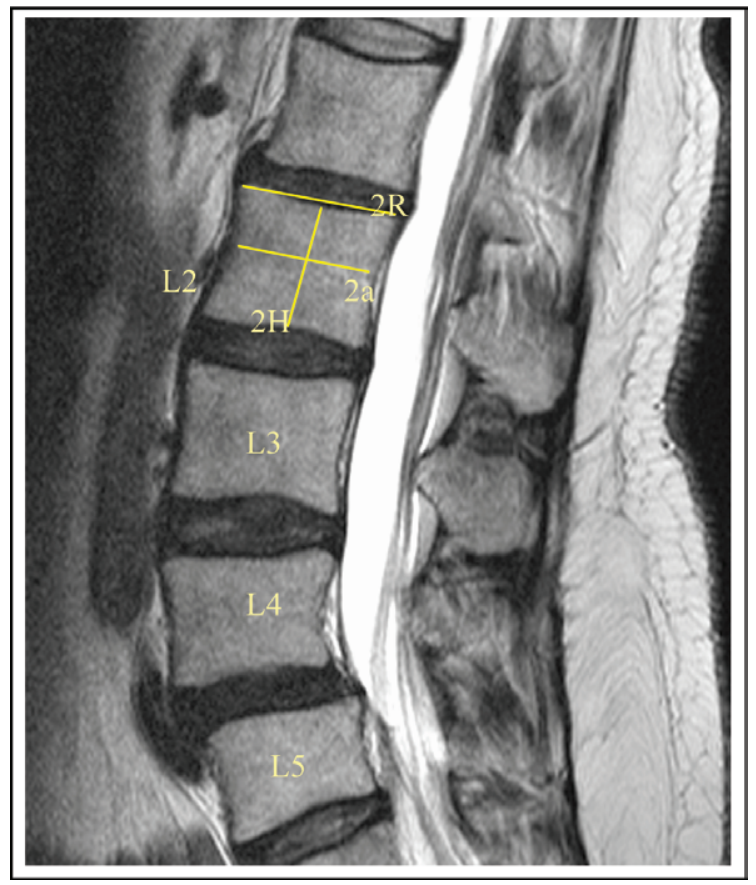

Figure 11: MRI of lumbar vertebrae. $H / R=0.7$ (average of L2-L5) and $a / R$ is 0.91 (average of L2-L5).

combined using the principle of linear superposition. For its optimal intrinsic design with respect to the hyperboloid shape parameter $\beta$, to sustain the combined loadings [i.e. compression from eqn (16), bending moment from eqn (26) and torsion from eqn (30)], we need to have

$$
\frac{\mathrm{d}}{\mathrm{d} \beta}[\text { Combined forces in the generators }]=0 .
$$

Hence from eqns (16), (26) and (30), we obtain

$$
\frac{\mathrm{d}}{\mathrm{d} \beta}\left(\frac{C}{2 n \cos \beta}+\frac{M}{n a \cos \beta}+\frac{T}{2 n a \sin \beta}\right)=0 .
$$

or

$$
\left(\frac{\sqrt{R^{2}-a^{2}}}{H}\right)^{3}\left(\frac{C}{2}+\frac{M}{a}\right)=\frac{T}{2 a} .
$$

Equation (32) gives the relationship between the applied loading and the geometry of the VB. Interestingly, the value of $a$ is not found in the literature [8, 9, 10]. However, for a specific set of values of $R$ and $H$ and functionally occurring ratios of the loading values $(C, M, T)$, the value of the hyperboloid shape parameter $a$ can be calculated from eqn (32), for the intrinsic design of the VB. In eqn (32), considering the representative values of $C=1000 \mathrm{~N}, M=T=3 \mathrm{Nm}$, along with 
$R=21.6 \mathrm{~mm}$ and $H=14.75 \mathrm{~mm}$, based on Zhou et al. [10] and Guo et al. [11], we obtain $a \approx$ $20.3 \mathrm{~mm}$; hence, from eqn (4), $\beta=26.5^{\circ}$.

Hence, the optimal lightweight, high-strength spinal VB geometry is given by $\beta=26.5^{\circ}$ with $a / R=0.939$ (for $H=14.75 \mathrm{~mm}$ ). The $a / R$ value of 0.91 measured from the lumbar vertebrae MRI scan shown in the Fig. 11 confirms our analysis. Thus, the intrinsic design of the VB hyperboloid geometry is such that it bears the combined loadings of compression, bending moment and torsion by minimizing the axial forces in the generators. In other words, it can sustain and transmit maximal values of the loadings with minimal amounts of material (because of all the loadings being transmitted as axial forces through the hyperboloid generators).

\section{CONCLUSION}

We have carried out an internal stress analysis of the hyperboloid VB under compression, bending moment and torsional loading. The analysis shows that all the loading states are transmitted by the VB generators as axial forces, thereby making it a high-strength structure with a high load-bearing capacity. Explicit expressions for these axial forces in the VB generators under compression, bending moment and torsional loading conditions are obtained (in terms of the VB geometrical parameters).

Minimization of the total axial force for the combined loadings gives the value of the hyperboloid shape parameter $\beta=26.5^{\circ}$, for which $a / R=0.939$, which closely matches the measured value of $a / R=0.91$ from the VB MRI. Therefore, for this value of $\beta$, the spinal VB can maximize its loadbearing capacity. Thus, we have demonstrated that the VB shape and material distribution are modelled by the loading to be an optimal high-strength and lightweight structure, in the same way as the femur's shape and material distribution are based on the stress trajectories (Fig. 1) due to the loading sustained by it.

\section{REFERENCES}

[1] Collins, M.W., Hunt, D.G. \& Atherton, M.A., Optimisation Mechanics in Nature, WIT Press: Southampton, 2004.

[2] Collins, M.W., Atherton, M.A. \& Bryant, J.A., Nature and Design, WIT Press: Southampton, 2005.

[3] Wolff, J., Das Gesetz der Transformation der Knochen, Hirschwald, 1892 (translated by P. Maquet \& R. Furlong (1986) as The Law of Bone Remodeling, Springer: Berlin, 1986).

[4] Harris, J.W. \& Stocker, H., Hyperboloid of revolution. Handbook of Mathematics and Computational Science, Springer-Verlag: New York, p. 112, 1998.

[5] Steinhaus, H., Mathematical Snapshots, Dover: New York, 1999.

[6] Raamachandran, J., Thin Shells: Theory \& Problems, Universities Press Limited: India, 1993.

[7] Elizabeth, C.E., Vladimir Shukhov and the invention of hyperboloid structures. Proc. of the 2005 Structures Congress and the 2005 Forensic Engineering Symposium, 2005.

[8] Nissan, M. \& Gilad, I., Dimensions of human lumbar vertebrae in the sagittal plane. Journal of Biomechanics, 19(9), pp. 753-758, 1986.

[9] Panjabi, M., Goel, V.K., Oxland, T., Takata, K., Duranceau, J., Krag, M. \& Price, M., Human lumbar vertebrae: quantitative three-dimensional anatomy. Spine, 17, pp. 298-306, 1992.

[10] Zhou, S.H., McCarthy, I.D., McGregor, A.H., Coombs, R.R.H. \& Hughes, S.P.F., Geometrical dimensions of the lower lumbar vertebrae-analysis of data from digitized CT images. European Spine Journal, 9, pp. 242-248, 2000.

[11] Guo, L.X., Teo, E.C. \& Qiu, T.Z., Prediction of biomechanical characteristics of intact and injured lower thoracic spine segment under different loads. Journal of Musculoskeletal Research, 8(2\&3), pp. 87-99, 2004. 